\title{
Implementation of Partial Synchronization of Different Chaotic Systems by Field Programmable Gate Array
}

\author{
Can Eroğlu \\ Department of Electrical and \\ Electronics Engineering \\ Izmir Institute of Technology \\ Izmir, Turkey 35430 \\ Email: caneroglu@iyte.edu.tr
}

\author{
F. Acar Savac1 \\ Department of Electrical and \\ Electronics Engineering \\ Izmir Institute of Technology \\ Izmir, Turkey 35430 \\ Email: acarsavaci@iyte.edu.tr
}

\begin{abstract}
In this study, the synchronization of the masterslave systems has been achieved and implemented on Field Programmable Gate Array (FPGA). In this paper, the master system and the slave system have been chosen as Lorenz and Rossler systems, respectively. The feedback control rule has been derived by feedback linearization method. By feedback linearization, the coordinate transformation has been achieved then the control command for synchronization has been obtained. In order to implement designed synchronized system, Matlab Simulink design of the system has been translated to Xilinx System Generator design to generate Very-High-Speed Integrated Circuits Hardware Description Language (VHDL) code which is used to produce bitstream file. By Xilinx Integrated Software Environment (ISE) program, VHDL code is converted to bitstream file which has been embedded into FPGA by Field Upgradeable Systems Environment (FUSE). Finally, the designed synchronized system has been observed on the HP 54540C oscilloscope.
\end{abstract}

\section{INTRODUCTION}

In the nonlinear science, control of chaotic systems have been studied recently owing to its particular importance in applications such as communications, physics etc. [1], [2]. There are several kinds of synchronizations such as generalized synchronization [3], complete synchronization [4], [5], partial synchronization [6], phase synchronization [7] and almost synchronization [8]. The pioneering work [5], has increased the interest in synchronization after having recently found many applications particularly in telecommunications [9], in mechanical systems [10] and in control theory [11]. Partial synchronization problem has been studied in populations of pulse-coupled oscillators [12].

This paper is organized as follows: In Section II the partial synchronization problem is defined and the feedback linearization method is explained. In Section III, as a case study Lorenz system is chosen as the master system and Rossler system is chosen as the slave system then by the suitable feedback, Rossler system is synchronized to Lorenz system. In Section IV, the proposed control system is simulated by Matlab Simulink then the simulated design is converted to Xilinx System Generator design and the designed synchronized system is implemented by the help of ISE and FUSE programs. Finally, in Section V, conclusions are presented.

\section{SyNCHRONIZATION PROBLEM}

In this section, the partial synchronization problem in the sense of exact synchronization and practical synchronization will be considered. The definitions given below describes exact synchronization, practical synchronization and partial synchronization, respectively.

Definition 2.1: It is said that two chaotic systems are exactly synchronized if the synchronization error, $e_{i}=x_{i}-y_{i}$ , exponentially converges to the origin. This implies that at a finite time $x_{i}=y_{i}$ [8].

Definition 2.2: It is said that two chaotic systems are practically synchronized if the trajectories of the synchronization error $e_{i}=x_{i}-y_{i}$ converges to a neighborhood around the origin. This implies that for all time $t \leq t^{*}$ the trajectories of the slave system are close to the master trajectories, i.e., $x_{i} \approx y_{i}$ [8].

Definition 2.3: It is said that two chaotic systems are partially synchronized if, at least, one of the states of the synchronization error system is either practically or exactly synchronized and only if, at least, one of the states of the synchronization error system is neither practically nor exactly synchronized [8].

The difference between master and slave system is called as an error system which can be constructed using the definition given below.

Definition 2.4: Let $\dot{\mathbf{x}}=\mathbf{F}_{M}(x)$ and $\dot{\mathbf{y}}=\mathbf{F}_{S}(y)+\mathbf{g}(y) u(y)$ be two chaotic systems in a manifold $\mathbf{M} \subset \mathrm{R}^{n} . \mathbf{F}_{M}, \mathbf{F}_{S}$ smooth vector fields with scalar output functions $s_{M}=h(x)$, $s_{S}=h(y)$ and $\mathbf{x}, \mathbf{y} \in \mathrm{R}^{n}$ and $\mathbf{g}(y) \in \mathrm{R}^{n}$ is a smooth input vector where subscripts $M$ and $S$ stands for the master and slave, respectively [4]. 


$$
\begin{aligned}
\dot{\mathbf{x}} & =\mathbf{F}_{M}(x), \\
\dot{\mathbf{e}} & =\mathbf{F}_{M}(x)-\mathbf{F}_{S}(x, e)-\mathbf{g}(x, e) u(x, e), \\
s_{e} & =h(x, e),
\end{aligned}
$$

where $s_{e}$ is the output of the synchronization error system and extended synchronization error system can be written in affine form as:

$$
\dot{\mathbf{X}}=\mathbf{F}(X)+\mathbf{G}(X) u(X)
$$

where $\mathbf{X}=[\mathbf{x}, \mathbf{e}]^{T}, \mathbf{F}(X)=\left[\mathbf{F}_{M}(x), \mathbf{F}_{M}(x)-\mathbf{F}_{S}(y)\right]^{T}$ and $\mathbf{G}(X)=[\mathbf{0},-\mathbf{g}(x, e)]^{T}$.

In order to achieve synchronization, synchronization error system in Eq. 2 should be stabilized around the equilibrium point $e^{*}=0$. The definitions and theorems given in the following sequel will be used to find the proper invertible transformation which will be used to derive the control command for synchronization.

Definition 2.5: System (4) is said to have relative degree $\rho$, $\rho \leq n$ at point $x_{0} \in \mathrm{R}^{n}$ with respect to the output

$$
s_{e}=h(x)
$$

if for any $x \in \Omega$ where $\Omega$ is some neighborhood of $x_{0}$, the following conditions are valid

(i) $L_{G} L_{F}^{k} h(x)=0, k=0,1, \ldots \rho-2, \forall x$ in a neighborhood of $x_{0}$ and $k<\rho-1$,

(ii) $L_{G} L_{F}^{\rho-1} h\left(x_{0}\right) \neq 0$.

Recall that $L_{\psi} \phi(x)=\sum_{i=1}^{n} \frac{\partial \phi}{\partial x_{i}} \psi(x)$ stands for the Lie derivative of the vector function $\phi$ along the vector field $\psi$. Relative degree $\rho$ is exactly equal to the number of times one has to differentiate the output in order to have the input explicitly appearing in the equation which describes the evolution of $s_{e}^{(r)}(t)$ in the neighborhood of $x_{0}$ [2], [13].

Theorem 2.6: System (4) is feedback linearizable in the neighborhood $\Omega$ of a point $x_{0} \in \mathrm{R}^{n}$ if and only if there exists a smooth scalar function $h(x)$ defined in $\Omega$ such that the relative degree $\rho$ of (3) and (4) is equal to $n$ [2].

Theorem 2.7: Consider the system (4). Suppose that there exist $2 n-\rho$ functions $\Phi_{i}(x, e)$ such that $L_{G} \Phi_{i}(x, e)=0$, $i=\rho+1, \ldots, 2 n$. This system is feedback linearizable at $(x, 0)$ if and only if there exists a function $h(x, e)$ such that (i) $<\partial h, a d_{F}^{k-1} \mathbf{G}>(x, e)=0$ for $k=1, \ldots, \rho-1 ; \rho>1$ and $(x, e)$ in a neighborhood $\Omega$ of $(x, 0)$,

(ii) $<\partial h, a d_{F}^{i} \mathbf{G}>(x, 0) \neq 0$ for $i=\rho, \ldots, n$ at $(x, 0)$,

where $\rho=d$ stands for the dimension of the tangent space and the accessibility distribution function $\mathbf{C}(x, e)$ can be expressed as $\mathbf{C}_{d}=\operatorname{span}\left\{a d_{F}^{d-1} \mathbf{G}\right\}$ where $a d_{F}=[\mathbf{F}, \mathbf{G}]$ and $a d_{F}^{d-1}=$
$[\mathbf{F},[\mathbf{F},[\ldots,[\mathbf{F}, \mathbf{G}], \ldots]]$,$] for d=1, \ldots, n$ where $[\mathbf{F}, \mathbf{G}]$ is called the Lie bracket of $\mathbf{F}$ and $\mathbf{G}$ [4].

Corollary 2.8: Two chaotic systems with the same order are completely synchronizable if and only if the dynamical error system is feedback linearizable at $(x, 0)$ [4].

Feedback linearization problem: The system in (4) is called feedback linearizable if there exist a smooth reversible change of coordinates $z=\Phi(x, e)$ and smooth transformation of the feedback [13], [14].

$$
u=\lambda(x, e)+\mu(x, e) v,
$$

where $v \in \mathrm{R}^{m}$ is the new control if the closed-loop is linear and then the resulting variables $z$ and $v$ satisfy linear dynamical system in the form of

$$
\begin{aligned}
\dot{\mathbf{z}} & =\mathbf{A z}+\mathbf{b v} \\
\mathbf{z} & =\Phi(x, e)=\left[h(x, e), L_{F} h(x, e), \ldots, L_{F}^{\rho-1} h(x, e)\right]^{T} \\
u & =\frac{1}{L_{G} L_{F}^{\rho-1} h(x, e)}\left(-L_{F}^{\rho} h(x, e)+v\right) \\
\lambda(x, e) & =\frac{-L_{F}^{\rho} h(x, e)}{L_{G} L_{F}^{\rho-1} h(x, e)} \\
\mu(x, e) & =\frac{1}{L_{G} L_{F}^{\rho-1} h(x, e)} \\
\nu & =\mathrm{K}_{i}\left(z_{i}-z_{i}^{*}\right)
\end{aligned}
$$

where $\mathrm{K}_{i}$ with $i=1, \ldots, \rho$ are the control gains and chosen in such a way that the closed-loop subsystem $\dot{z}$ converges to the origin and $z_{i}^{*} \mathrm{~s}$ are the coordinates of the stabilization point. In order to achieve complete synchronization $z_{i}^{*}$ s are set to zero.

\section{SynChRONIZATION OF ChAOTIC Systems WiTH DIFFERENT MODEL}

Lorenz system [15] is chosen as the master system and its state equations can be written as follows :

$$
\begin{aligned}
\dot{x_{1}} & =\alpha\left(x_{2}-x_{1}\right) \\
\dot{x_{2}} & =\beta x_{1}-x_{2}-x_{1} x_{3} \\
\dot{x_{3}} & =x_{1} x_{2}-\gamma x_{3}
\end{aligned}
$$

where $\alpha=10, \beta=28$, and $\gamma=8 / 3$. Rossler system [16] is chosen as the slave system and is described as:

$$
\begin{aligned}
& \dot{y_{1}}=-\left(y_{2}+y_{3}\right)+g_{1}(y) u \\
& \dot{y_{2}}=y_{1}+\theta y_{2}+g_{2}(y) u \\
& \dot{y_{3}}=\delta+y_{3}\left(y_{1}-\phi\right)+g_{3}(y) u
\end{aligned}
$$

where $g_{1}(y), \quad g_{2}(y)$ and $g_{3}(y)$ are control inputs and $\theta=0.2, \delta=0.2, \phi=5.7$. The extended synchronization error system for coupled Lorenz-Rossler system can be written by calculating the error system as: 
$C_{3}(x, 0)$ can be written as:

$$
\begin{aligned}
& \dot{e_{1}}=\alpha\left(x_{2}-x_{1}\right)+\left(x_{2}-e_{2}\right)+\left(x_{3}-e_{3}\right)-g_{1}(x, e) u \\
& \dot{e_{2}}=\beta x_{1}-x_{2}-x_{1} x_{3}-\left(x_{1}-e_{1}\right)-\theta\left(x_{2}-e_{2}\right)-g_{2}(x, e) u \\
& \dot{e_{3}}=x_{1} x_{2}-\gamma x_{3}-\delta-\left(x_{3}-e_{3}\right)\left[\left(x_{1}-e_{1}\right)-\phi\right]-g_{3}(x, e) u
\end{aligned}
$$

After having found error sytem, the extended synchronization error system can be written as:

$$
\begin{aligned}
\dot{x_{1}} & =\alpha\left(x_{2}-x_{1}\right)
\end{aligned}
$$

then $\mathbf{F}(X)$ and $\mathbf{G}(X)$ can be found as in Eq. (4)

$$
\begin{gathered}
\mathbf{F}=\left[\begin{array}{c}
\alpha\left(x_{2}-x_{1}\right) \\
\beta x_{1}-x_{2}-x_{1} x_{3} \\
x_{1} x_{2}-\gamma x_{3} \\
\alpha\left(x_{2}-x_{1}\right)+\left(x_{2}-e_{2}\right)+\left(x_{3}-e_{3}\right) \\
\beta x_{1}-x_{2}-x_{1} x_{3}-\left(x_{1}-e_{1}\right)-\theta\left(x_{2}-e_{2}\right) \\
x_{1} x_{2}-\gamma x_{3}-\delta-\left(x_{3}-e_{3}\right)\left[\left(x_{1}-e_{1}\right)-\phi\right]
\end{array}\right] \\
\mathbf{G}=\left[\begin{array}{c}
0 \\
0 \\
0 \\
-g_{1}(x, e) \\
-g_{2}(x, e) \\
-g_{3}(x, e)
\end{array}\right]
\end{gathered}
$$$$
\mathbf{C}_{3}(x, 0)=\operatorname{span}\left\{\left[\begin{array}{ccc}
0 & 0 & 0 \\
0 & 0 & 0 \\
0 & 0 & 0 \\
0 & -1 & \theta \\
-1 & \theta & 1-\theta^{2} \\
0 & 0 & x_{3}
\end{array}\right]\right\}
$$

In order to derive the control command, we need to determine the dimension of the tangent space $d$ which is generated by the corresponding distribution so the conditions of Theorem 2.7 must be satisfied. The dimension of the tangent space $d$ is determined to be equal to 2 since the conditions below have been satisfied.

$$
\begin{aligned}
-\frac{\partial h}{\partial e_{2}} & =0 \\
-\frac{\partial h}{\partial e_{1}}+\theta \frac{\partial h}{\partial e_{2}} & \neq 0
\end{aligned}
$$

For this case $h(x, e)=e_{1}$ can be chosen as an output function which satisfies conditions of Theorem 2.7 then (9) $d=\rho=\operatorname{Dim}\left(\mathbf{C}_{3}(x, 0)\right)=2, \forall x \in \mathrm{R}^{3}$ and considering Theorem 2.6, to have feedback linearizable system for $\rho=2$, the conditions below must be satisfied

$$
\begin{aligned}
L_{G} h(x, e) & =0 \\
L_{G} L_{F} h(x, e) & \neq 0
\end{aligned}
$$

The corresponding accessibility distribution function $\mathbf{C}_{3}(x, e)$ can be calculated where $\mathbf{C}_{3}(x, e)=$ $\operatorname{span}\left\{\mathbf{G}, \operatorname{ad}_{F} \mathbf{G}, a d_{F}^{2} \mathbf{G}\right\}$. Let $\mathbf{G}$ be defined as

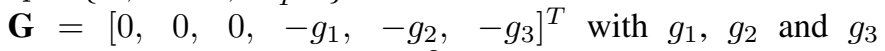
constants, then $a d_{F} \mathbf{G}$ and $a d_{F}^{2} \mathbf{G}$ will be calculated to obtain $\mathbf{C}_{3}(x, e)$.

$$
\begin{aligned}
& \mathbf{C}_{3}(x, e)=\operatorname{span}\left\{\left[\begin{array}{ccc}
0 & 0 & 0 \\
0 & 0 & 0 \\
0 & 0 & 0 \\
-g_{1} & -g_{2}-g_{3} & b^{*} \\
-g_{2} & g_{1}+\theta g_{2} & c^{*} \\
-g_{3} & a^{*} & d^{*}
\end{array}\right]\right\} \\
& a^{*}=g_{1}\left(x_{3}-e_{3}\right)+g_{3}\left(x_{1}-e_{1}-\phi\right) \\
& b^{*}=g_{1}\left(1+x_{3}-e_{3}\right)+\theta g_{2}+g_{3}\left(x_{1}-e_{1}-\phi\right) \\
& c^{*}=-\theta g_{1}+g_{2}\left(1-\theta^{2}\right)+g_{3} \\
& d^{*}=-g_{1}\left(x_{1}-e_{1}-\phi\right)\left(x_{3}-e_{3}+1\right)+g_{2}\left(x_{3}-e_{3}\right) \\
& +g_{3}\left[1+x_{3}-e_{3}-\left(x_{1}-e_{1}-\phi\right)^{2}\right]
\end{aligned}
$$

For simplicity by setting $g_{1}=0, g_{2}=1$ and $g_{3}=0$ then then the transformation can be found as

$$
\begin{aligned}
z_{1} & =h(x, e)=e_{1} \\
z_{2} & =L_{F} h(x, e)=\dot{e}_{1}=\alpha\left(x_{2}-x_{1}\right)+\left(x_{2}-e_{2}\right)+\left(x_{3}-e_{3}\right)
\end{aligned}
$$

The complementary functions which should satisfy such that $L_{G} \Phi_{i}(x, e)=0, i=2 n-\rho, \ldots 2 n$ can be chosen as

$$
\begin{aligned}
& z_{3}=w_{1} \\
& z_{4}=x_{1} \\
& z_{5}=x_{2} \\
& z_{6}=x_{3}
\end{aligned}
$$

Eqs. (14)and (15) constitute an invertible transformation around $(x, 0)$ which means that there is a proper control command $u(x, e)$ such that the slave system trajectory tracks the master system trajectory. The transformed system can then be written as follows:

$$
\begin{aligned}
\dot{z_{1}} & =z_{2} \\
\dot{z_{2}} & =L_{F}^{2} h(x, e)+L_{G} L_{F} u(x, e) \\
\dot{z_{3}} & =\dot{w_{1}} \\
\dot{z_{4}} & =\dot{x_{1}} \\
\dot{z_{5}} & =\dot{x_{2}} \\
\dot{z_{6}} & =\dot{x_{3}}
\end{aligned}
$$


where the control command can be found as:

$$
\begin{aligned}
& u(x, e)=\frac{1}{L_{G} L_{F} h(x, e)}\left(-L_{F}^{2} h(x, e)+\kappa_{1} z_{1}+\kappa_{2} z_{2}\right) \\
& u(x, e)=\alpha \dot{x_{1}}-(1+\alpha) \dot{x_{2}}-\dot{x_{3}}+\dot{e_{2}}+\dot{e_{3}}+\kappa_{1} e_{1}+\kappa_{2} \dot{e_{1}}
\end{aligned}
$$

where $\dot{x_{1}}, \dot{x_{2}}, \dot{x_{3}}, \dot{e_{1}}, \dot{e_{2}}$ and $\dot{e_{3}}$ can be written in terms of $x_{1}, x_{2}, x_{3}, e_{1}, e_{2}$ and $e_{3}$.

\section{Simulation AND implementation RESUlts of THE DESIGNED SYNCHRONIZED SYSTEM}

After simulating the designed system, in order to generate VHDL code, Xilinx System Generator blocks can be used. System Generator is a very useful tool since Simulink design is easily converted to System Generator blocks and it works under Matlab as a toolbox which provides to generate VHDL code of the designed system. Then, this VHDL code is used to produce bitstream file by using Xilinx ISE program. At the last step, bitstream file is embedded into FPGA by FUSE program.

The results of designed system can be observed on HP54540 scope as in Fig. (1). The state $e_{1}(t)=x_{1}(t)-y_{1}(t)$ is exactly synchronized while the state $e_{2}(t)=x_{2}(t)-y_{2}(t)$ is practically synchronized and the state $e_{3}(t)=x_{3}(t)-y_{3}(t)$ is not synchronized. Therefore, Lorenz system is said to be partially synchronized with Rossler system.

\section{CONCLUSION}

The control system, Lorenz's attractor and Rossler's attractor have been embedded into FPGA. The controller has been designed to synchronize Rossler's attractor to Lorenz's attractor by feedback linearization method and partial synchronization of Lorenz system with Rossler system has been achieved.

\section{REFERENCES}

[1] R. Femat, R. Jauregui-Ortiz and G. Solis-Perales, "A Chaos-Based Communication Scheme via Robust Asymptotic Feedback", IEEE Transactions on Circuits and Systems I: Fundemental Theory and Applications vol. 48, no. 10, pp. 1161-1169, 2001.

[2] A. L. Fradkov, Cybernetical Physics: From Control of Chaos to Quantum Control, Springer-Verlag, 2007.

[3] V. S. Afraimovich, N. N. Verichev and M. I. Rabinovich, "Stochastic synchronization of oscillations in dissipative systems", Radiophysics and Quantum Electronics vol. 29, pp. 795-803, 1987.

[4] R. Femat and G. Solis-Perales, Robust Synchronization of Chaotic Systems via Feedback, Springer-Verlag, 2008.

[5] L. M. Pecora and T. L. Carrol, "Synchronization in chaotic systems", Physics Review Letter vol. 64, no. 08, pp. 821-824, 1991.

[6] Yu Maistrenko and O. Popovych, "On strong and weak chaotic partial synchronization", International Journal of Bifurcation and Chaos, vol. 10, pp. 179-203, 2000.

[7] M. G. Rosenblum, A. S. Pikovsky and J. Kurths, "Phase synchronization in driven and coupled chaotic oscillators", Physica D, vol. 104, pp. 219238, 1997.

[8] R. Femat and G. Solis-Perales, "On the Chaos Synchronization Phenomena", Physics Letters A, vol. 262, no. 01, pp. 50-60, 1999.

[9] A. Abel and W. Schwarz, "Chaos communications - principles, schemes and system analysis", Proceedings of the IEEE, vol. 90, no. 05, pp. 691710, 2002.

[10] I. I. Blekhman, P. S. Landa and M. G. Rosenblum, "Synchronization and chaotization in interacting dynamical systems", Applied Mechanics Review, vol. 48, pp. 733-752, 1995.
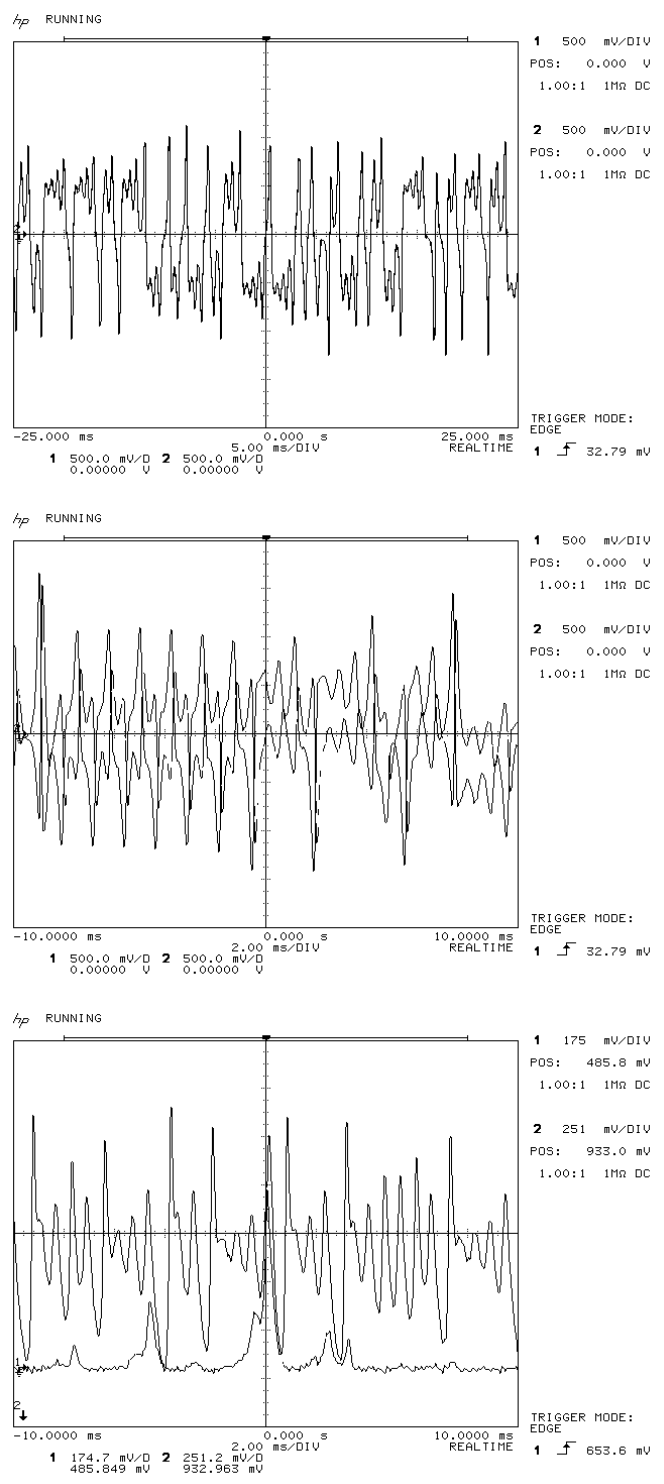

Fig. 1. Partial Practical Synchronization of Lorenz system with Rossler system. From the top to the bottom Channel 1 represetns $x_{1}(t)$ and Channel 2 represents $y_{1}(t)$; Channel 1 represetns $x_{2}(t)$ and Channel 2 represents $y_{2}(t)$; Channel 1 represetns $x_{3}(t)$ and Channel 2 represents $y_{3}(t)$, respectively.

[11] H. Nijmeijer, "A dynamical control view on synchronization" Physica $D$, vol. 154, pp. 219-228, 2001.

[12] C. V. Vreeswijk "Partial synchronization in populations of pulse-coupled oscillators" Physical Review E, vol. 54, pp. 5522-5537, 1996.

[13] M. Vidyasagar, Nonlinear System Analysis, $2^{\text {nd }}$ ed., Prentice-Hall International, 1993.

[14] B. R. Andrievskii , A. L. Fradkov, "Control of Chaos: Methods and Applications. I. Methods", Automation and Remote Control, vol. 64, no. 05, pp. 673-713, 2003.

[15] N. E. Lorenz, "Deterministic Nonperiodic Flow", Journal of Atmospheric Science, vol. 20, no. 02, pp. 130-141, 1963.

[16] O. E. Rössler, "An Equation for Continuous Chaos", Physics Letter 35A, vol. 57 , no. 05 , pp. 397-398, 1976. 